\title{
Multiobjective Optimization of Power Control for CDMA Systems
}

\author{
Chang-Yi Yang \\ Department of Computer Science and Information \\ Engineering \\ National Penghu University of Science and Technology \\ Penghu, Taiwan \\ e-mail: cyyang@npu.edu.tw
}

\author{
Hong-Yao Chen \\ Department of Computer Science and Information \\ Engineering \\ National Penghu University of Science and Technology \\ Penghu, Taiwan \\ e-mail: kevin123821@yahoo.com.tw
}

\begin{abstract}
In this study, a multi-objective (MO) optimization method is proposed for power control design in CDMA systems. With a shadow system and an $H_{\infty}$ filter to compensate for the round-trip delay, the proposed power control scheme can simply adjust transmission power to achieve the best compromise between several objectives, such as minimization of SINR deviation, minimization of power consumption, and minimization of the system outage. A simulation result is given to confirm the performance of the proposed power control scheme.
\end{abstract}

Keywords-Multi-objective (MO) optimization, shadow system, $H_{\infty}$ filter

\section{INTRODUCTION}

Over the last few decades, the code division multiple access (CDMA) technique has attracted a great attention in the field of wireless mobile communications due to its potential to support a large number of simultaneous users than the conventional access schemes [1],[2]. However, there are some problems in CDMA systems, such as multiple access interference (MAI) and near-far problem [3]. Power control is an essential tool to cope with the above problems.

Several power control schemes have been designed for each user to track a target signal-to-interference-and-noise ratio (SINR) without consideration of SINRs of other users in a cell or multi-cell [4],[5]. They adjust the transmission power to minimize the deviation between the received SINR and the target SINR. However, the target SINR can not be achieved at any arbitrary high power level because power consumption is also an important problem in CDMA communication systems. From the network's perspective, cutting power consumption not only prolongs the life of the battery, but also decreases the MAI to other users in the network. Besides, when the user can not achieve the minimum required SINR, the system performance is degraded and outage occurs. The communication link is then blocked out. Therefore, minimizing the target SINR, power consumption, and system outage should be considered simultaneously in the power control design of CDMA communication systems.

Recently, the utility-based power control schemes in [6],[7] consider multiple objectives rather than single objective. When multiple objectives are considered, one of the most rapidly developing methodologies is the multi- objective (MO) optimization [8] which integrates several objectives with different weights for resource management. In [9], an MO optimization scheme is presented for solving the distributed power control problem by making a tradeoff in MO targets. However, it is not robust and is unsuitable for severe channel fading and round-trip delay. Meanwhile, the weights of the MO optimization are decided beforehand by the designer and are therefore a subjective factor.

In this paper, an optimal power controller is designed based on the MO optimization with a shadow system and an $H_{\infty}$ filter (SS- $H_{\infty}$ ). The minimizations of SINR deviation, power consumption, and system outage are considered at the same time. The Step method (STEM) for multicriteria decision analysis [8] is introduced to decide the weights of objectives. This controller can adjust transmission power for the best compromise between MO targets. Besides, the shadow system is constructed to simulate the power control update command through a transmission channel but without the effect of round-trip delay, i.e., to compensate for the round-trip delay. Since the shadow system does not come in contact with channel environments directly, it is necessary to estimate the channel state and channel interferences for the shadow system. The $H_{\infty}$ filter based on robust theory can be employed to estimate channel state regardless of the statistics of channel interferences, as long as the total power of the channel interferences is bounded [10]. Therefore, the proposed scheme can combat the round-trip delay and estimate the transmission power in the power control loop. The estimation result is useful for the MO optimization. Combining the MO optimization with the SS- $H_{\infty}$ scheme, the proposed power control scheme can overcome channel interferences and round-trip delay.

\section{SYSTEM MODEL}

In the CDMA cellular system, the closed-loop power control in log scale (i.e., dB scale) is shown in Fig. 1 [11]. The received SINR $y_{i}(k)$ is compared with the target SINR $y_{i}^{\text {tar }}(k)$ so that the tracking error $e_{i}(k)$

$$
e_{i}(k)=y_{i}^{\text {tar }}-y_{i}(k)
$$

is obtained. The SINR deviation $e_{i}(k)$ will be fed into the power control circuit to generate the power control update 
command $u_{i}(k)$. The quantizer transfers the update command from analog $u_{i}(k)$ to digital bits $\hat{u}_{i}(k)$ based on a quantization scalar. $\hat{u}_{i}(k)$ is then transmitted via the downlink channel with downlink delay $d_{i, 1}$.

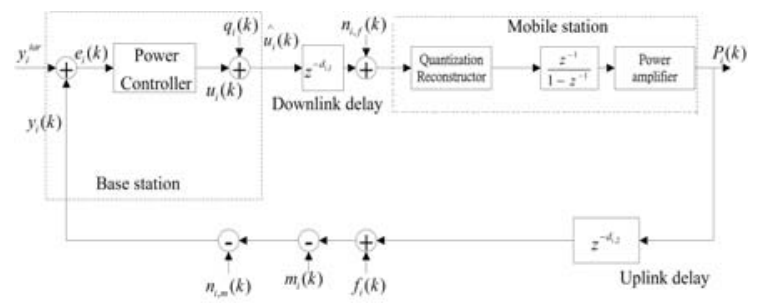

Figure 1. Closed-loop power control for user $i$ in the CDMA communication system.

At the mobile station, the quantization reconstructor is used to recover the quantized power control update command $\hat{u}_{i}(k)$ and then feeds it into the power multiplier to generate power $P_{i}(k)$, which $P_{i}(k)$ is limited to the interval [ $P_{i}^{\min }, P_{i}^{\max }$ ] because of the physical limitations in the power amplifier. The power $P_{i}(k)$ is delivered through the uplink channel with an uplink delay $d_{i, 2}$.

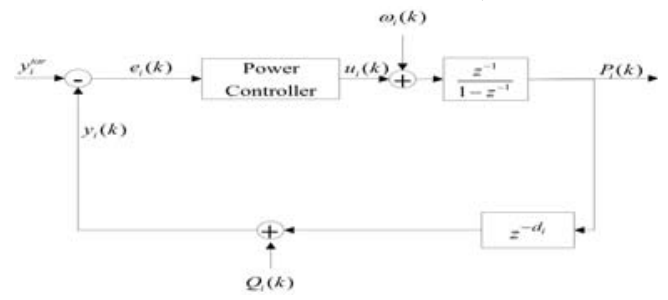

Figure 2. Equivalent simple closed-loop power control system for user i.

For simplicity, the closed-loop power control system can be transformed from the topology shown in Fig. 1 to the equivalent system given in Fig. 2 [10], where the round-trip delay $d_{i}$ is the sum of downlink delay and uplink delay $d_{i}=d_{i, 1}+d_{i, 2}$.

Thus, the mathematical model of power control system can be denoted as

$$
\begin{aligned}
& P_{i}(k)=P_{i}(k-1)+u_{i}(k-1)+w_{i}(k-1) \\
& P_{i}^{\min } \leq P_{i}(k) \leq P_{i}^{\max } \\
& y_{i}(k)=P_{i}\left(k-d_{i}\right)+Q_{i}(k)
\end{aligned}
$$

The definitions of $w_{i}(k)$ and $Q_{i}(k)$ please refer to [7]. Because the transmission power $P_{i}(k)$ suffers the roundtrip delay $d_{i}$, the power control update command $u_{i}(k)$ also contains $d_{i}$. Therefore, the power control update command can not adjust the transmission power in real time to achieve the target SINR precisely. A power control scheme will be developed in this paper to compensate for the round-trip delay and provide the MO optimization so that the optimal target SINR tracking, the minimal power consumption, and the minimal system outage can be achieved simultaneously.

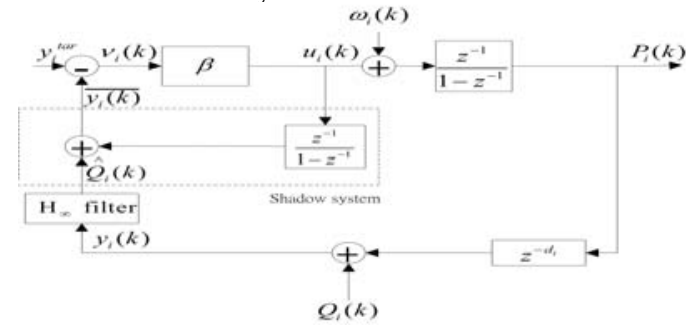

Figure 3. Power control system with an shadow system scheme.

\section{TIME DELAY COMPENSATION AND TRANSMISSION POWER ESTIMATION USING SHADOW SySTEM AND $H_{\infty}$ FILTER}

In order to compensate for the round-trip delay $d_{i}$, a shadow system is constructed to simulate the power control update command $u_{i}(k)$ through a transmission channel, but without the effect of $d_{i}$ (See Fig. 3). In the shadow system, $\frac{z^{-1}}{1-z^{-1}}$ is used to model the transformation from the power update command $u_{i}(k)$ to the transmission power $P_{i}(k)$. Moreover, the downlink interference $w_{i}(k)$ and the uplink interference $Q_{i}(k)$ have to be considered in the shadow system. Since $w_{i}(k)$ is a small interference, it can be compensated through an adaptive law [12]. Therefore, $w_{i}(k)$ can be ignored in the shadow system. However, the uplink interference $Q_{i}(k)$ has to be estimated (represented as $\hat{Q}_{i}(k)$ ) and used in the shadow system for time-delay compensation. The $H_{\infty}$ filter is suitable for estimating $Q_{i}(k)$ because it does not require any knowledge of the statistics of $Q_{i}(k)$. From (2), the estimation of $P_{i}(k)$ (represented as $\left.\hat{P}_{i}(k)\right)$ can be obtained by $H_{\infty}$ filter. The residue can be an estimate of $Q_{i}(k)$ (represented as $\left.\hat{Q}_{i}(k)\right)$.

Through the shadow system, the feedback signals $\bar{y}_{i}(k)$ and $v_{i}(k)$ in Fig. 3 are described as:

$$
\begin{aligned}
& \bar{y}_{i}(k)=\frac{u_{i}(k) z^{-1}}{1-z^{-1}}+\hat{Q}_{i}(k) \\
& v_{i}(k)=y_{i}^{\text {tar }}-\bar{y}_{i}(k)=y_{i}^{\text {tar }}-\frac{u_{i}(k) z^{-1}}{1-z^{-1}}-\hat{Q}_{i}(k)
\end{aligned}
$$


It is obvious that the feedback signal $v_{i}(k)$ in (3) is independent of the round-trip delay. The power control update command $u_{i}(k)$ is generated by:

$$
u_{i}(k)=\beta v_{i}(k)
$$

where $\beta$ is the power control gain.

\section{Multiobjective Optimization POWER CONTROL DESIGN}

According to the user objective and the network dynamics, a power control policy will follow three objectives to construct a power control cost function:

O1). minimization of SINR deviation: The SINR deviation $e_{i}(k)$ is described in (1).

O2). minimization of power consumption: Since the transmission power $P_{i}(k)$ can not be measured at the base station, the estimated transmission power $\hat{P}_{i}(k)$ from $H_{\infty}$ filter is used. The power consumption function $g_{i}(k)$ is formulated as follows [9]:

$$
g_{i}(k)=\alpha\left(P_{i}(k)-P_{i}^{\text {min }}\right)
$$

If the power consumption is considered, $\alpha>0$ is set in (10).

O3). minimization of system outage: The system outage occurs when the received SINR $y_{i}(k)$ is below the minimum required SINR $y_{i}^{\min }$. Therefore, the transmission power is adjusted so that the received SINR is as close as possible to the minimum required SINR.

$$
o_{i}(k)=y_{i}^{\mathrm{min}}-y_{i}(k)
$$

In Fig. 3, the transmission power $P_{i}(k)$ is adjusted according to power control update command $u_{i}(k)=\beta v_{i}(k)$. Therefore, an optimal power control gain $\beta$ is chosen to adjust transmission power to minimize the three objectives. The MO power control problem is formulated as the following constrained optimization

$$
\begin{array}{ll}
\min _{\beta \in[\underline{\beta}, \bar{\beta}]}\left\{\left|e_{i}(k)\right|,\left|g_{i}(k)\right|,\left|o_{i}(k)\right|\right\}= \\
& \min _{\beta \in[\underline{\beta}, \bar{\beta}]}\left\{\left|y_{i}^{\text {tar }}-y_{i}(k)\right|,\left|\alpha\left(P_{i}(k)-P_{i}^{\text {min }}\right)\right|,\left|y_{i}^{\text {min }}-y_{i}(k)\right|\right\} \\
\text { s.t } \quad & P_{i}(k)=P_{i}(k-1)+u_{i}(k-1)+w_{i}(k-1) \\
& P_{i}^{\text {min }} \leq P_{i}(k) \leq P_{i}^{\max }
\end{array}
$$

where the power control game $\beta$ is assumed to be in the region of $[\underline{\beta}, \bar{\beta}]$ because power control should be within the limit range to stabilize the whole close-loop power control system.

Through the weighting matrices, the deviation function $E_{i}(k)$ for MO problem in (7) can be constructed as follows

$E_{i}(k)=\lambda_{i, 1}(k)\left|y_{i}^{\text {tar }}-y_{i}(k)\right|$

$$
+\lambda_{i, 2}(k)\left|\alpha\left(\hat{P}_{i}(k)-P_{i}^{\text {min }}\right)\right|+\lambda_{i, 3}(k)\left|y_{i}^{\text {min }}-y_{i}(k)\right|
$$

subject to

$$
\begin{aligned}
\hat{P}_{i}(k) & =\hat{P}_{i}(k-1)+\beta v_{i}(k-1) \\
& +K_{i}(k-1)\left(y_{i}(k-1)-\hat{P}_{i}\left(k-d_{i}-1\right)\right), \\
P_{i}^{\min } & \leq \hat{P}_{i}(k) \leq P_{i}^{\max }
\end{aligned}
$$

where the weighting factors $\lambda_{i, 1}(k), \lambda_{i, 1}(k)$, and $\lambda_{i, 1}(k)$

in (8) satisfy the following constraint

$$
\begin{aligned}
& 0 \leq \lambda_{i, 1}(k) \leq 1 \\
& 0 \leq \lambda_{i, 2}(k) \leq 1 \\
& 0 \leq \lambda_{i, 3}(k) \leq 1 \\
& \lambda_{i, 1}(k)+\lambda_{i, 2}(k)+\lambda_{i, 3}(k)=1
\end{aligned}
$$

The specification of weighting factors becomes important for the MO power control. However, it is difficult to assign or know $\lambda_{i, 1}(k), \lambda_{i, 1}(k)$, and $\lambda_{i, 1}(k)$ precisely in every time step. The Step Method (STEM) is one of the first iterative methods developed for MO optimization problems [8]. The decision maker with this method allows the values of some acceptable objective functions to increase so that the unacceptable functions can have lower values. In this study, based on the concept of the STEM, the weighting factors can be assigned as follows.

$$
\begin{aligned}
& \lambda_{i, 1}(k)=\frac{a_{i, 1}(k)}{a_{i, 1}(k)+a_{i, 2}(k)+a_{i, 3}(k)} \\
& \lambda_{i, 2}(k)=\frac{a_{i, 2}(k)}{a_{i, 1}(k)+a_{i, 2}(k)+a_{i, 3}(k)} \\
& \lambda_{i, 3}(k)=\frac{a_{i, 3}(k)}{a_{i, 1}(k)+a_{i, 2}(k)+a_{i, 3}(k)}
\end{aligned}
$$

where the relative important coefficients $a_{i, j}(k), j=1,2,3$, are given by

$$
\begin{aligned}
& a_{i, 1}(k)=\left[\left|\frac{y_{i}^{\text {tar }}-y_{i}(k)}{y_{i}^{\text {tar }}}\right|\right]\left[\left(y_{i}^{\text {tar }}\right)^{2}+y_{i}(k)^{2}\right]^{-1 / 2} \\
& a_{i, 2}(k)=\left[\left|\frac{\alpha\left(\hat{P}_{i}(k)-P_{i}^{\text {min }}\right)}{P_{i}^{\text {min }}}\right|\right]\left[\left(P_{i}^{\text {m in }}\right)^{2}+\hat{P}_{i}(k)^{2}\right]^{-1 / 2} \\
& a_{i, 3}(k)=\left[\left|\frac{y_{i}^{\text {min }}-y_{i}(k)}{y_{i}^{\text {min }}}\right|\right]\left[\left(y_{i}^{\text {min }}\right)^{2}+y_{i}(k)^{2}\right]^{-1 / 2}
\end{aligned}
$$

Finally, if we choose $\beta$ within $0<\beta<2$, the closedloop power control system can achieve robust stability [7].

\section{SimULATION}

An optimal $\beta$ should be chosen from the intersection region $[\underline{\beta}, \bar{\beta}] \cap(0,2)$ to minimize the power control cost function $J_{i}(\beta)$. In general, it is not easy to find a closedform solution for an optimal $\beta \in[\underline{\beta}, \bar{\beta}] \cap(0,2)$ to satisfy the constrained optimization problem. However, with the help of a Monte Carlo simulation, the corresponding optimal $\beta$ can be approached. The example is simulated for 50 runs. Each run performs the simulation at 10000 sample points.The base station assigns a specified data rate for each mobile unit before transmission. The velocity of each mobile unit is set to $140 \mathrm{~km} / \mathrm{hr}$. Simulation results for the mean values of power control cost function $J(\beta)$ versus $\beta$ are displayed in Fig. 4. According to the results at 
different data rates and different $\alpha$ in Fig. 4. If $\alpha$ is 0.01 , $\beta$ is selected as $0.2,0.8,0.2$ for low data rate (data rate $=$ $9.6 \mathrm{kHz}$ ), median data rate (data rate $=153.6 \mathrm{kHz}$ ), and high data rate (data rate $=921.6 \mathrm{kHz}$ ), respectively. If $\alpha$ is 0.05 , $\beta$ is selected as $0.1,0.7,0.2$ for low data rate, median data rate, and high data rate, respectively. Therefore, based on different data rates and $\alpha$, an adequate control gain in the power control loop can be chosen to achieve the MO optimization.

\section{CONCLUSION}

In this study, an MO optimization is considered to achieve the minimization of SINR deviation, power consumption, and system outage simultaneously. Besides, an SS- $H_{\infty}$ scheme is proposed to estimate the transmission power and uplink interference to compensate for the effect of round-trip delay. It can provide a robust power tracking performance in a wireless environment with round-trip delay and channel interference. From the simulation, several values for $\beta$ are obtained for different data rates. To analyze the effects of the round-trip delay, data rate, and outage probability is our next step.

\section{ACKNOWLEDGMENT}

This work is partly supported by the National Science Council (NSC) in Taiwan under grant NSC101-2221-E-346009.

\section{REFERENCES}

[1] K. S. Gilhousen, I. M. Jacobs, R. Padovani, A. J. Viterhi, L. A. Weaver, and C. E. Wheatley, "On the capacity of a cellular CDMA system,” IEEE Trans. Veh. Technol., vol. 40, pp. 303-312, May 1991.
[2] F. Simpson and J. M. Holtzman, "Direct sequence CDMA power control, interleaving, and coding," IEEE J. Select. Areas Commun., vol. 11, no. 7, pp. 1085-1094, Sep. 1993.

[3] D. C. Chen, B. J. Sheu, and W. C. Young, "A CDMA communication detector with robust near-far resistance using paralleled array processors," IEEE Circuits and Systems for Video Technology, vol. 7, pp. 654-662, Aug. 2002.

[4] G. J. Foschini and Z. Miljanic, "A simple ditributed autonomous power control algorithm and its convergence," IEEE Trans. Veh. Technol., vol. 42, no. 4, pp. 641-646, Nov. 1993.

[5] C. C. Chai, Y. H. C. Y.Lu, and T. T. Tjhung, "A unified framework for transmitter power control in cellular radio systems,” ETRI J., vol. 26, no. 5, pp. 341-349, 2004.

[6] N. Feng, S. Mau, and G. Pottie, "Pricing and power control for joint network-centric and user-centric radio resource management,” IEEE Trans. Commun., vol. 52, pp. 1547-1557, Sep. 2004.

[7] B. S. Chen, C. Y. Yang, and S. Y. Li, "Adaptive two-loop power tracking control in CDMA systems with the utility optimization," IEEE Trans. Wireless Communications, vol. 7, no. 4, pp. 1358-1367, April 2008.

[8] H. F. Wang, Multicriteria Decision Analysis From Certainty To Uncertainty. Ting Lung Book Co., 2004.

[9] M. Elmusrati, R. Jantti, and H. N. Koivo, "Multiobjective distributed power control algorithm for CDMA wireless communication systems," IEEE Trans. Veh. Technol., vol. 56, no. 2, pp. 779-788, Mar. 2007.

[10] B. K. Lee, Y. H. Chen, and B. S. Chen, "Robust $H 1$ power control for CDMA cellular communication systems,” IEEE Trans. Signal Processing, vol. 54, no. 10, pp. 3947-3956, Oct. 2006.

[11] R. Patachaianand and K. Sandrasegaran, "Performance study of new adaptive power control algorithm," in in International Symposium on Communiation and Information Technologies, May 2007.

[12] H. J. Su and E. Geraniotis, "Adaptive close-loop power control with quantized feedback and loop-filtering," IEEE Trans. Commun., vol. 1, no. 1, pp. 76-86, Jan. 2002.
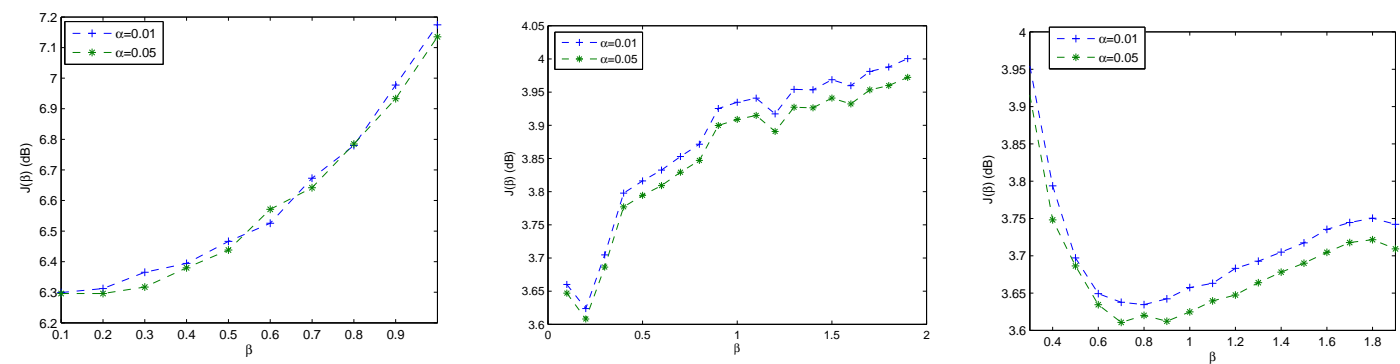

Figure 4. The mean values of power control cost function $J(\beta)$ versus power control gain $\beta$ at different data rates in the proposed power control scheme (a) for low data rate $9.6 \mathrm{kHz}$. (b) for median data rate $153.6 \mathrm{kHz}$. (c) for high data rate $921.6 \mathrm{kHz}$. 